\title{
Cellular fatty acid synthase is required for late stages of HIV-1 replication
}

\author{
Manjusha M. Kulkarni ${ }^{1 \dagger}$, Annette N. Ratcliff ${ }^{4,5 \dagger}$, Menakshi Bhat ${ }^{4}$, Yazan Alwarawrah², Philip Hughes², \\ Jesus Arcos ${ }^{1}$, David Loiselle ${ }^{2}$, Jordi B. Torrelles ${ }^{1,6}$, Nicholas T. Funderburg ${ }^{3}$, Timothy A. Haystead $2^{2^{*} \neq}$ \\ and Jesse J. Kwiek ${ }^{4^{*} \neq}$
}

\begin{abstract}
Background: Like all viruses, HIV-1 relies on host systems to replicate. The human purinome consists of approximately two thousand proteins that bind and use purines such as ATP, NADH, and NADPH. By virtue of their purine binding pockets, purinome proteins are highly druggable, and many existing drugs target purine-using enzymes. Leveraging a protein affinity media that uses the purine-binding pocket to capture the entire purinome, we sought to define purine-binding proteins regulated by HIV-1 infection.

Results: Using purinome capture media, we observed that HIV-1 infection increases intracellular levels of fatty acid synthase (FASN), a NADPH-using enzyme critical to the synthesis of de novo fatty acids. siRNA mediated knockdown of FASN reduced HIV-1 particle production by $80 \%$, and treatment of tissue culture cells or primary PBMCs with Fasnall, a newly described selective FASN inhibitor, reduced HIV-1 virion production by $90 \%\left(\mathrm{EC}_{50}=213 \mathrm{nM}\right)$. Despite the requirement of FASN for nascent virion production, FASN activity was not required for intracellular Gag protein production, indicating that FASN dependent de novo fatty acid biosynthesis contributes to a late step of HIV-1 replication.

Conclusions: Here we show that HIV-1 replication both increases FASN levels and requires host FASN activity. We also report that Fasnall, a novel FASN inhibitor that demonstrates anti-tumor activity in vivo, is a potent and efficacious antiviral, blocking HIV-1 replication in both tissue culture and primary cell models of HIV-1 replication. In adults, most fatty acids are obtained exogenously from the diet, thus making FASN a plausible candidate for pharmacological intervention. In conclusion, we hypothesize that FASN is a novel host dependency factor and that inhibition of FASN activity has the potential to be exploited as an antiretroviral strategy.
\end{abstract}

Keywords: Human immunodeficiency virus type 1, Host-virus interaction, Fatty acid synthase, Antiviral pharmacology, Fasnall

\section{Background}

Viruses repurpose host cellular synthetic and metabolic pathways to produce progeny. Using large-scale CRISPR

\footnotetext{
*Correspondence: timothy.haystead@duke.edu; kwiek.2@osu.edu ${ }^{\dagger}$ Manjusha M. Kulkarni and Annette N. Ratcliff contributed equally to this work

FTimothy A. Haystead and Jesse J. Kwiek contributed equally to this work

${ }^{2}$ Department of Pharmacology and Cancer Biology, Duke University

School of Medicine, C118 LSRC, Box 3813, Durham, NC 27710, USA

${ }^{4}$ Department of Microbiology, Center for Retrovirus Research, The Ohio

State University, 476 Biological Sciences Building, 484 W. 12th Avenue,

Columbus, OH 43210, USA

Full list of author information is available at the end of the article
}

[1] or siRNA-based screens, several groups have identified host proteins required for replication of West Nile virus (WNV) [2], dengue virus (DENV) [3], hepatitis C virus (HCV) [4], influenza [5], and human immunodeficiency virus type-1 (HIV-1) [6-10]. These studies established the range of host proteins that modulate viral replication, and by extension, they also highlight the potential to develop antiviral drugs that target host proteins $[7,11]$. Maraviroc, which targets host CCR5 molecules and blocks HIV-1 replication, is the prototypical host directed antiviral drug [11]. Recently, our laboratories and others have shown that inhibitors of the 
chaperone proteins Hsp70 and Hsp90 inhibit Chikungunya virus (CHIKV) and dengue fever virus (DENV) replication in cell and animal models [12-14]. Since host therapeutic targets evolve more slowly than viral therapeutic targets, antiviral therapy targeting host proteins would likely impose a high barrier to drug resistance. Further, if several viruses require the same host pathway, the potential exists to develop a pan-antiviral drug. The current challenge is to identify a host target that when inhibited, it limits viral replication while simultaneously not harming the host.

Here we report that HIV-1 infection increases host fatty acid synthase (FASN) levels, and a decrease in FASN activity attenuates HIV replication during a late stage of its replication cycle. We also report that Fasnall, a novel FASN inhibitor with anti-tumor activity [15] potently reduces HIV-1 production with minimal effects on cellular viability.

\section{Results}

\section{Identification of purine-binding proteins regulated} by HIV-1 infection

We used an unbiased, functional chemoproteomic screen $[16,17]$ to define purine-binding proteins regulated by HIV-1 infection. HeLa-derived TZM-bl cells were infected with HIV-1, lysed $48 \mathrm{~h}$ post infection, and incubated with the purinome-capture resin. After extensive washing, bound proteins were competed off the purinome-capture resin with ATP. HIV-infection increased the recovery of several human proteins from the purinome-binding resin, including FASN, heat-shock protein 90 (HSP90), and others (Fig. 1). HSP90 is a validated cancer target and its role in HIV-1 replication has been reported elsewhere [18-20]. We focused on FASN (see band 3, Fig. 1), owing to its specialized, well-defined cellular function (de novo fatty acid synthesis), limited tissue expression [21], and previously reported association with flavivirus replication [22-24].

\section{HIV-1 regulates host FASN}

To validate the purinome-capture of FASN, we measured FASN expression in TZM-bl cells 24 and $48 \mathrm{~h}$ post HIV-1 infection. FASN mRNA levels, normalized to $18 \mathrm{~S}$ rRNA, did not change following HIV-1 infection, suggesting FASN regulation in TZM-bl cells occurs post-transcriptionally (Fig. 2a). Western blotting with a FASN-specific antibody confirmed that HIV-1 infection increases FASN levels twofold to fivefold, as early as $24 \mathrm{~h}$ post infection; similar increases in FASN protein levels were also observed following HIV-1 infection of SupT1 and THP-1 cells (Fig. 2b). To determine if HIV-infection regulates FASN activity, we quantified intracellular fatty acid (FA) levels in TZM-bl cells with or with HIV-1. Our results

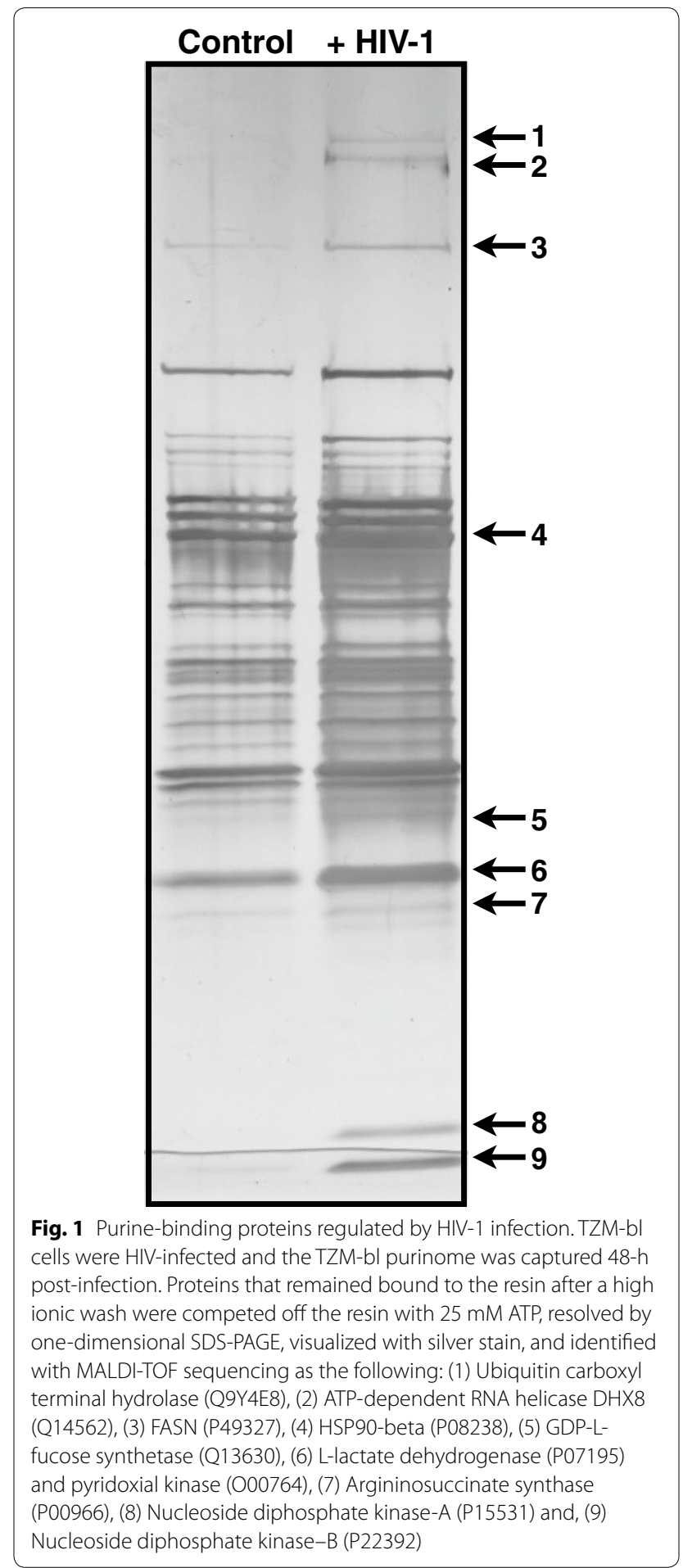

show that $48 \mathrm{~h}$ post infection, HIV-1 increased intracellular palmitic, oleic, and stearic acid levels (Fig. 2c). Because FA extractions were performed from an equivalent number of cells, it is unlikely that increased FA levels were due to differences in cellular biomass. 

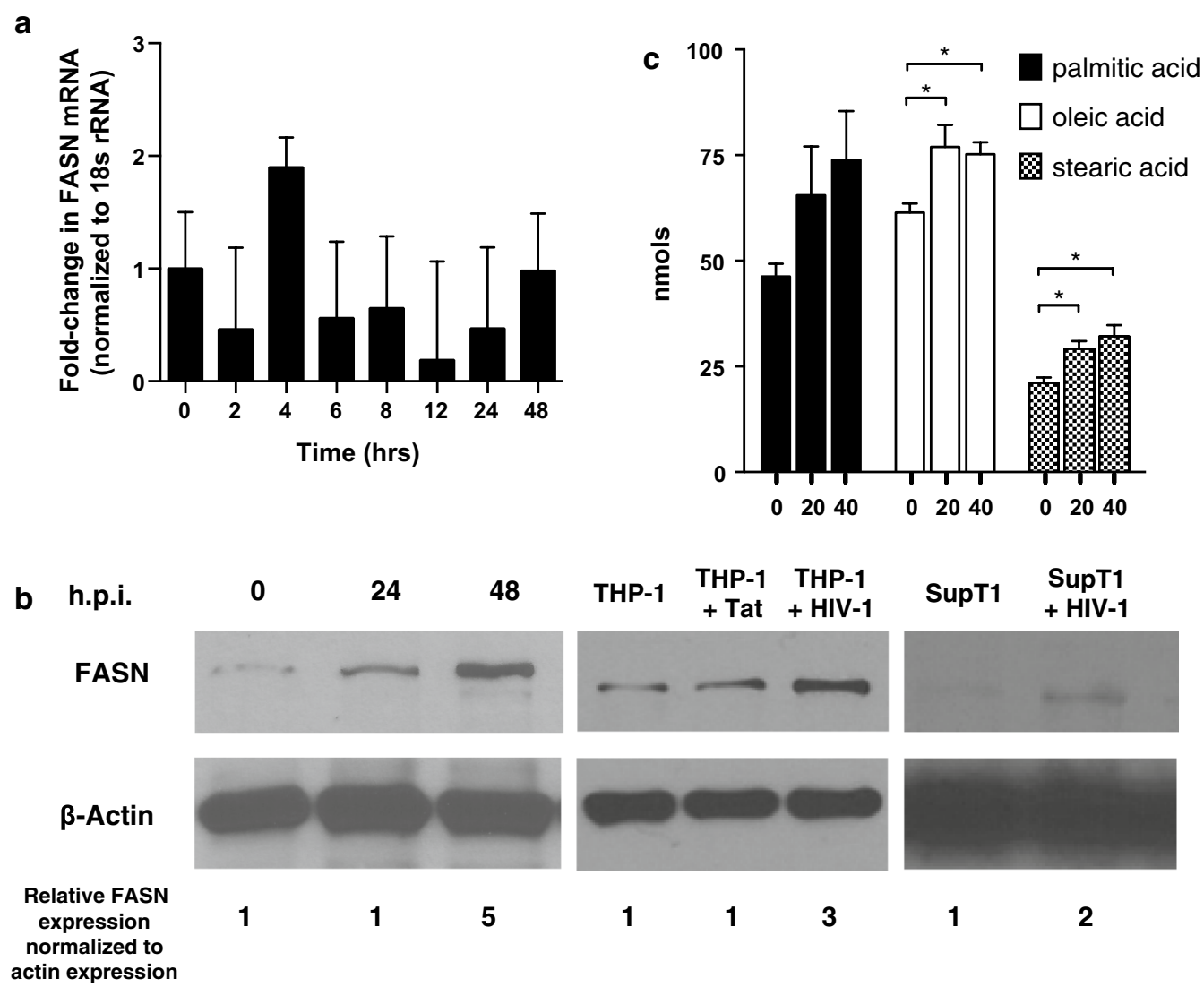

Fig. 2 HIV-1 infection of TZM-bl cells does not alter FASN mRNA levels but it does increase FASN protein and fatty acid levels. a FASN mRNA levels, normalized to $18 \mathrm{~S}$ rRNA levels, following infection with HIV-1 ${ }_{\mathrm{NL} 43}$ for the indicated number of hours post infection (h.p.i). Normalized mRNA levels at $4 \mathrm{~h}$ post infection are not significantly different than normalized mRNA levels at time $0, p=0.2$, Student's $t$ test. Data are representative of two independent experiments. b Western blot analysis of FASN and actin protein levels in TZM-bl cell lysates [left], THP-1 lysates following pcTAT transfection or HIV-1 infection [middle], or SupT1 lysates [right] with or without HIV-1 infection. ImageJ software was used to calculate relative FASN expression normalized to actin expression. $\mathbf{c}$ Fatty acid quantification by gas chromatography was performed on extracts of TZM-bl cells following $48 \mathrm{~h}$ of infection with 0,20 , or $40 \mathrm{ng}$ p24/mL of HIV-1. The data presented are mean values ( \pm SD) from four independent experiments. ${ }^{*}$ indicates $p<0.05$ (Student's $t$ test). h.p.i $=$ hours post infection

FASN is a $272 \mathrm{kDa}$, multifunctional, cytosolic enzyme that uses NADPH to condense acetyl-CoA and malonylCoA into palmitate [25]. It has been shown that viral infections can change subcellular localization of FASN; for example, Dengue [22] infection causes FASN to relocalize to a perinuclear space, and Vaccinia virus infection relocalizes FASN to the mitochondria [26]. To determine if HIV-1 infection also causes FASN relocalization, we used immunofluorescence to monitor FASN distribution in HIV-1 infected TZM-bl cells. Although the intensity of FASN staining increased following HIV-1 infection, redistribution of FASN to a perinuclear space, lysosomes (Fig. 3a), mitochondria (Fig. 3b), or the endoplasmic reticulum (Fig. 3c) was not observed. Thus, similar to HCV [27], HIV-1 infection does not cause intracellular FASN redistribution.

\section{Fasnall is a novel FASN inhibitor that reduces HIV-1 replication}

We recently reported the discovery of a thiophenopyrimidine molecule-Fasnall-that potently and selectively inhibits FASN activity in vitro and also demonstrates anti-tumor activity in vivo [15]. To determine if Fasnall blocks HIV-1 replication, we infected TZM-bl with HIV-1 and $48 \mathrm{~h}$ post infection measured extracellular p24 levels as surrogate measure of HIV-1 replication. In this model, Fasnall potently inhibited HIV-1 p24 production with an $\mathrm{EC}_{50}$ of $213 \mathrm{nM}(95 \% \mathrm{CI}$ 93-487 $\mathrm{nM})$ and an estimated cellular toxicity $\left(\mathrm{TC}_{50}\right)$ of $10 \mu \mathrm{M}$ (Fig. 4a), resulting in an antiviral index $\left(\mathrm{TC}_{50} /\right.$ $\mathrm{EC}_{50}$ ) of 47. To determine if Fasnall blocked HIV-1 in activated T-cells, we measured p24 production from HIV-1 infected primary PBMCs in the presence or 

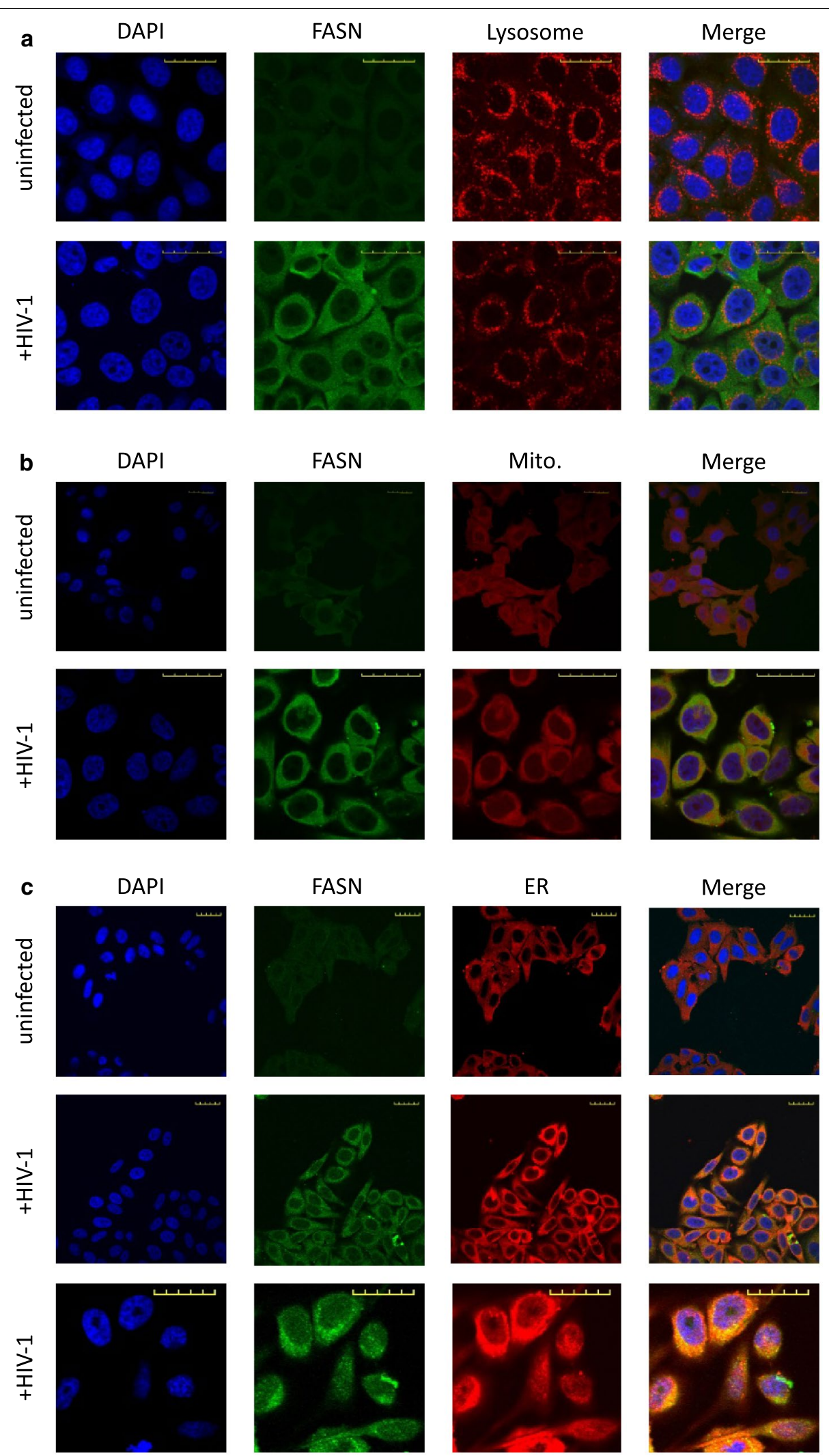

Fig. 3 Incubation of TZM-bl cells with HIV-1 increases intensity of FASN staining but does not change FASN subcellular localization. In all panels, FASN is labeled green and the nucleus is colored blue (DAPI). Red color denotes a lysosome (CD63), b mitochondria [Mito] (Mitotracker), c endoplasmic reticulum [ER] (calreticulin). Yellow scale bar equals $20 \mu \mathrm{m}$. Data are representative of two independent experiments 

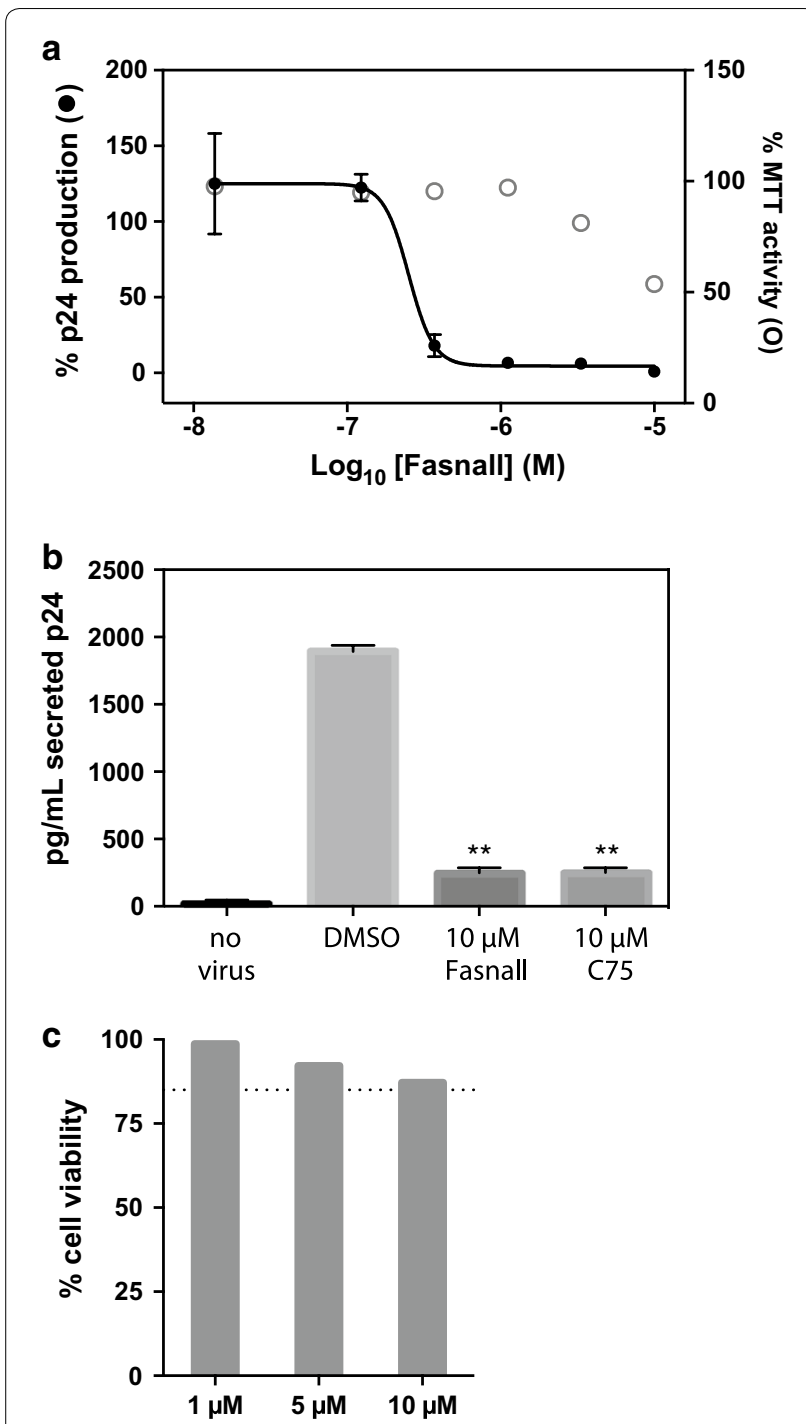

[Fasnall]

Fig. 4 Fasnall inhibits HIV-1 replication. a Extracellular p24 levels in TZM-bl cells $48 \mathrm{~h}$ post infection (black dots $\pm S D, n=3$, black line), without significant effects on TZM-bl cell viability (i.e. mean MTT-activity; open circles, $\mathrm{n}=3$ ) b Fasnall and C75, each at $10 \mu \mathrm{M}$, significantly reduce HIV-1 replication in primary PBMC, as measured by $\mathrm{p} 24$ production. The data presented are mean values $( \pm S D)$ from three independent experiments. ${ }^{*}$ indicates $p<0.0001$, treatment versus DMSO-treated control (Students $t$ test), c PBMC viability after treatment with Fasnall as measured by propidium iodide staining (dotted line drawn at 85\%)

absence of $10 \mu \mathrm{M}$ Fasnall. In this physiological relevant model of HIV-1 replication, Fasnall reduced HIV-1 p24 production approximately tenfold (Fig. 4b), with minimal effects on cell viability (Fig. 4c). Moreover, when PBMCs were treated with C75, a commercially available FASN inhibitor, similar reductions in extracellular p24 levels were observed (Fig. 4b). Thus, FASN activity is required for efficient HIV-1 replication in primary PBMCs.

\section{FASN activity modulates the late stages of HIV-1 replication}

To further confirm that the inhibitory effect of Fasnall and C75 was mediated by FASN, we transfected TZMbl cells with either FASN-specific or non-targeting siRNAs (abbreviated NT). Following siRNA mediated FASN knockdown (Fig. 5a), cells were HIV-infected for $48 \mathrm{~h}$, and extracellular p24 levels were again measured. Similar to the Fasnall results, siRNA-mediated FASN reduction reduced the levels of HIV-1 p24 released into the culture supernatant by $77 \%$, compared to non-targeting siRNA control cells (Fig. 5b, left). Despite this decrease in culture supernatant p24 levels, siRNA-mediated FASN knockdown did not significantly reduce intracellular p24 levels, measured by ELISA (Fig. 5b, right), suggesting that HIV-1 replication uses FASN activity during a late step in HIV-1 replication (e.g. protein trafficking, virion assembly, or virion release from the cell). Anti-Gag western blot of HIV-infected, FASN-knockdown cells indicates similar levels of intracellular p55 and p24 (Fig. 5c).

To test further the hypothesis that HIV-1 replication requires FASN activity during the late stages of viral replication, we transfected TZM-bl or SupT1 (data not shown) cells with a HIV-1 provirus (pNL43) in the presence or absence of Fasnall or C75. Similar to siRNAbased FASN knockdown, Fasnall-based inhibition of FASN did not reduce intracellular Gag levels (Fig. 6a) but did significantly reduce HIV-1 p24 particle deposition into culture medium, as measured by 24 production (Fig. 6b). Fasnall and C75 similarly reduced the number of infectious HIV-1 particles (Fig. 6c). Thus, FASN inhibition reduces nascent HIV-1 virion production without reducing HIV-1 protein synthesis.

\section{Discussion}

Here we show that HIV-1 infection increases FASN expression, and using both siRNA and pharmacological tools, we show that FASN inhibition blocks a late stage of HIV-1 replication. We also report that Fasnall, a nextgeneration FASN inhibitor with in vivo antitumor activity, has potent anti-HIV activity in both cell-culture and PBMC-based models of HIV-1 replication. From these findings, we hypothesize that FASN is a host-dependency factor.

Several siRNA-based studies of HIV-1 host dependency factors have been published (reviewed in [7]), none of which reported FASN as a host dependency factor. Many enveloped viruses, including cytomegalovirus (CMV), DENV, Epstein-Barr virus, HCV, HBV, influenza virus, Respiratory syncytial virus (RSV), and West Nile 

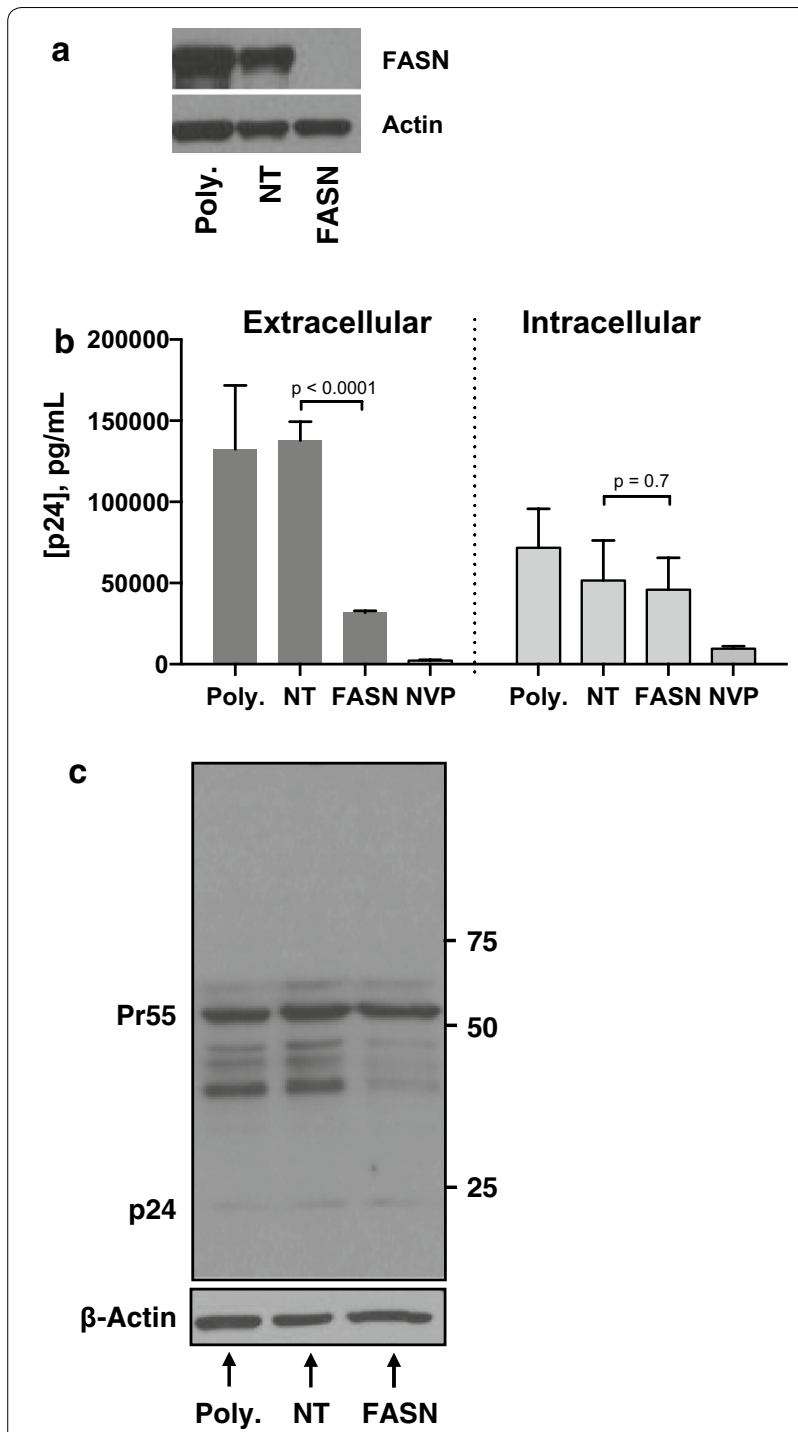

Fig. 5 FASN knockdown reduces HIV-1 particle production without affecting intracellular Gag production. a FASN immunoblot confirms reduction in endogenous FASN levels in TZM-bl cells. b Extracellular (left) and intracellular (right) p24 levels following siRNA-mediated FASN knockdown in TZM-bl cells infected with HIV-1 for $48 \mathrm{~h}$. Bars represent mean p24 \pm SD, quantified using a commercial ELISA, $n=3, p$-values generated with student's $t$ test. c anti-Gag western blot of lysates from HIV-infected cells with or without FASN knockdown. Molecular weight markers (kD) indicated in right margin. Poly = polymer (transfection) control, NT = nontargeting siRNA (siRNA control), FASN $=$ FASN-targeted siRNAs, NVP $=0.4 \mu \mathrm{M}$ nevirapine

Virus, also require host FASN activity to replicate efficiently [22-24, 28-30]. Building on these results, we now show that similar to many other enveloped viruses, efficient HIV-1 replication requires host FASN activity.

FASN expression is highly regulated in cells and can change ten-fold (or more) in response to physiological stresses such a starvation, lactation or pathological states [25]. FASN up-regulation has been observed in breast cancer, melanoma, and hepatocellular carcinoma [31]. Our in vitro results show that HIV-1 infection increases intracellular FASN levels in both TZM-bl and SupT1 cells. These results complement findings from others that showed HIV-1 infection increased FASN levels in both CEMx174 [32] and RH9 T-cells [33]. Similar changes in FASN levels have also been observed in people living with HIV-1; specifically, in a study of 191 people living with HIV-1, Aragones et al. [34] showed that people living with HIV-1 not taking antiretroviral therapy (ART) had elevated serum FASN levels compared to both HIVnegative people and people living with HIV-1 on ART. Thus, our results are consistent with previous in vitro and in vivo studies that correlated HIV-1 infection with increased FASN levels.

FASN catalyzes the complete synthesis of palmitate from acetyl-CoA and malonyl-CoA into long-chain saturated FAs. FASN is a multifunctional enzyme that synthesizes FA chains two-carbons at a time, each donated from malonyl-CoA. The final product, palmitic acid $(16: 0)$ is then released, where it can be metabolized further by $\beta$-oxidation, into myristic acid (14:0), or other long chain FAs [25]. Long chain FAs are essential components of lipid bilayers, store energy liberated by $\beta$-oxidation, and FAs can be covalently attached to proteins as a means to control protein subcellular localization [35]. The results presented here show that FASN activity is required for efficient HIV-replication, but it remains unclear how de novo synthesized FA are used by HIV-1. Potential mechanisms (Fig. 7) include the following: (1) provision of FA's used for ATP production and energy homeostasis, (2) creation of lipid micro domains associated with HIV-1 budding, (3) generation of fattyacyl adducts (e.g. palmitate or myristate) for post-translational modification (PTM) of Env, Gag, Nef, or host proteins required for HIV-1 replication, or (4) replenishment of phospholipids to regenerate the lipid bilayer lost during viral budding. Available evidence suggests that FASN-dependent cancers likely regulate de novo FA biosynthesis to produce lipids for membrane synthesis and energy production (i.e. Fig. 7, mechanism 4) [36]. Vaccinia virus infection has been shown to cause FASN to relocalize to mitochondria, likely for energy homeostasis (i.e. Fig. 7, mechanism 1) [26]. Flaviviruses such as HCV and Dengue virus likely use FASN/FA to rearrange intracellular membranes to replicate their genomes on membranous webs, which is not represented in Fig. 7 [22, 23]. When FASN activity is inhibited in the context of HIV-1 infection, HIV-1 Gag is produced but viral particles are not released into the culture supernatant. Based on this observation, we expect hypotheses 2 and 3 offer the 

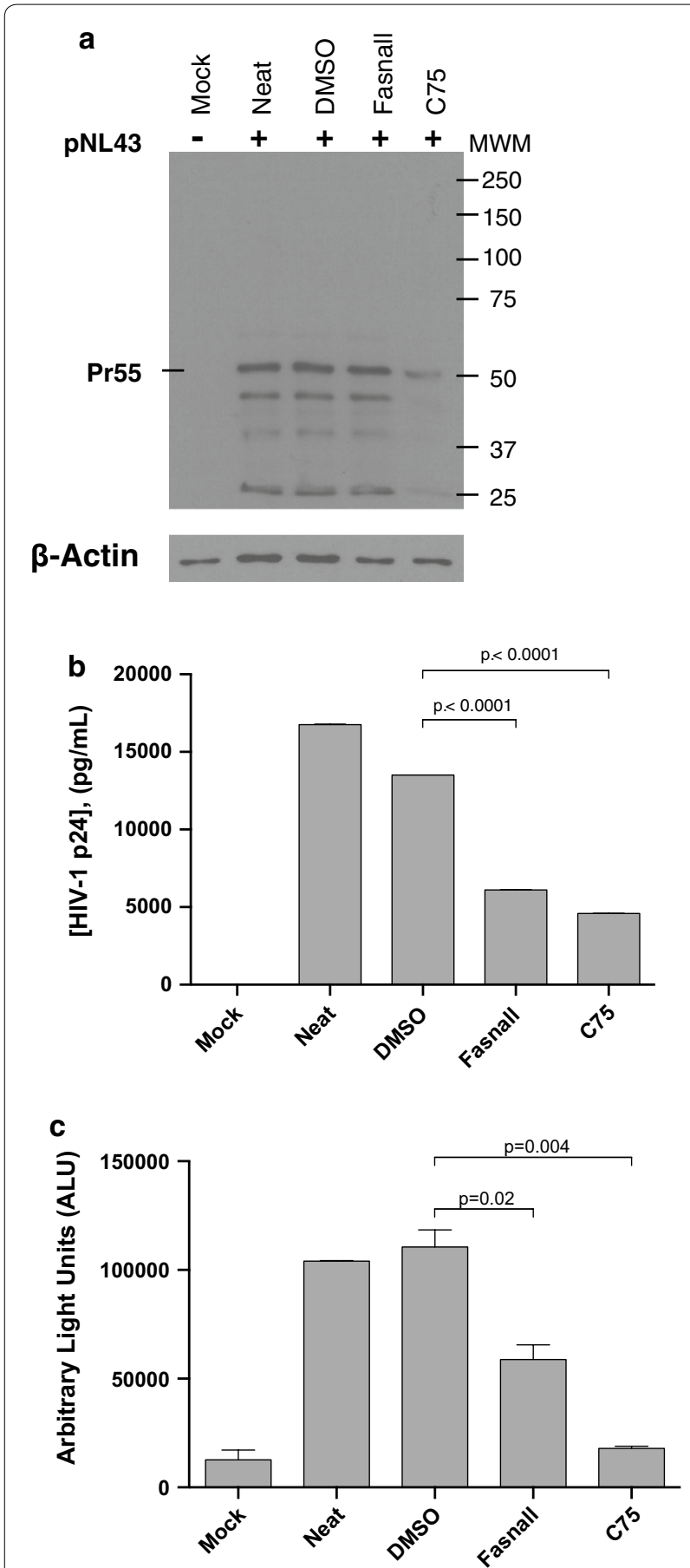

Fig. 6 FASN inhibition disrupts a late step in the HIV-1 replication cycle. a TZM-bl cells were transfected with pNL4-3 provirus plasmid for $48 \mathrm{~h}$ in the presence or absence of $10 \mu \mathrm{M}$ Fasnall or $10 \mu \mathrm{M}$ C75 or DMSO (0.01\%). Intracellular expression of HIV-1 proteins was monitored by HIVIG-western blot, and $\beta$-actin was used as loading control. b Supernatant associated virion production was monitored using a p24 ELISA. c $48 \mathrm{~h}$ post-transfection, cell culture supernatants were removed and incubated with fresh TZM-bl (indicator) cells for an additional 48 h. Values expressed as the mean \pm standard deviation, and are representative of three independent experiments. p-values were generated with student's $t$ test. Molecular weight markers $(\mathrm{kD})$ indicated in right margin most plausible mechanisms by which HIV-1 leverages FASN activity: to generate FA to create cholesterol-rich lipid micro domains that promote viral budding [37-40], or to generate fatty-acyl adducts required for viral protein function [41-44]. Regarding hypothesis 3 (protein acylation), several HIV-1 proteins contain fatty-acyl modifications, including palmitoylation (Env, Nef), and myristoylation (Gag). Env has been shown to be palmitoylated, although the literature provides conflicting evidence regarding whether Env palmitoylation is required for Env function [45] or not [46]. Our results indicate that reduction of FASN activity affects HIV-1 replication post-translation, and this result is reminiscent of the phenotype observed when the $\mathrm{N}$-terminal glycine of Gag is changed to an alanine [41, 47]. Specifically, it has been observed that Gag myristoylation is required for GagGag multimerization [42], and mutation of the glycine at amino acid position two, which is required for matrix myristoylation, abolishes viral particle release [47]. Incubation of cells with alternative substrates of $\mathrm{N}$-myristoyl transferases has been shown to block HIV-1 virus-like particle (VLP) production [43]. Thus, a plausible hypothesis states that HIV-1 stimulates de novo FA synthesis to produce myristate, which is required for Gag function.

FASN is considered a front-line drug target for the treatment of metabolic syndromes, cancer, and $\mathrm{HCV}$ [48-50], and data from this report suggest that FASN could be targeted in anti-HIV therapy. In adults, most normal tissues obtain FA exogenously from diet, and as a result, most cells have limited de novo FA biosynthesis and express FASN at very low levels [21]. Although studies in mice indicate that FASN is required for embryonic development [51], liver or macrophage-specific FASN knockout mice are viable $[52,53]$. We recently identified a new FASN inhibitor (Fasnall) that showed potent antitumor activity in a MMTV-Neu model of breast cancer [15]. In that study, we reported that in mice, a $15 \mathrm{mg} / \mathrm{mL}$ intraperitoneal (IP) injection of Fasnall did not alter animal weight, and it also did not alter blood cell counts or markers of liver and kidney function [15]. At this same dose, we also observed that Fasnall achieved a peak plasma concentration of $27 \mu \mathrm{M}$, which is 150 times greater than the $\mathrm{EC}_{95}$ value of Fasnall against HIV-1 replication (in TZM-bl cells). As several enveloped viruses require FASN activity, we suggest that Fasnall could be a useful starting point in the development of a pan-antiviral therapy.

\section{Conclusions}

Here we have shown that HIV-1 infection increases FASN expression. Using siRNA and small molecule FASN inhibitors, we have also shown that FASN activity contributes to HIV-1 replication during a late state in the 


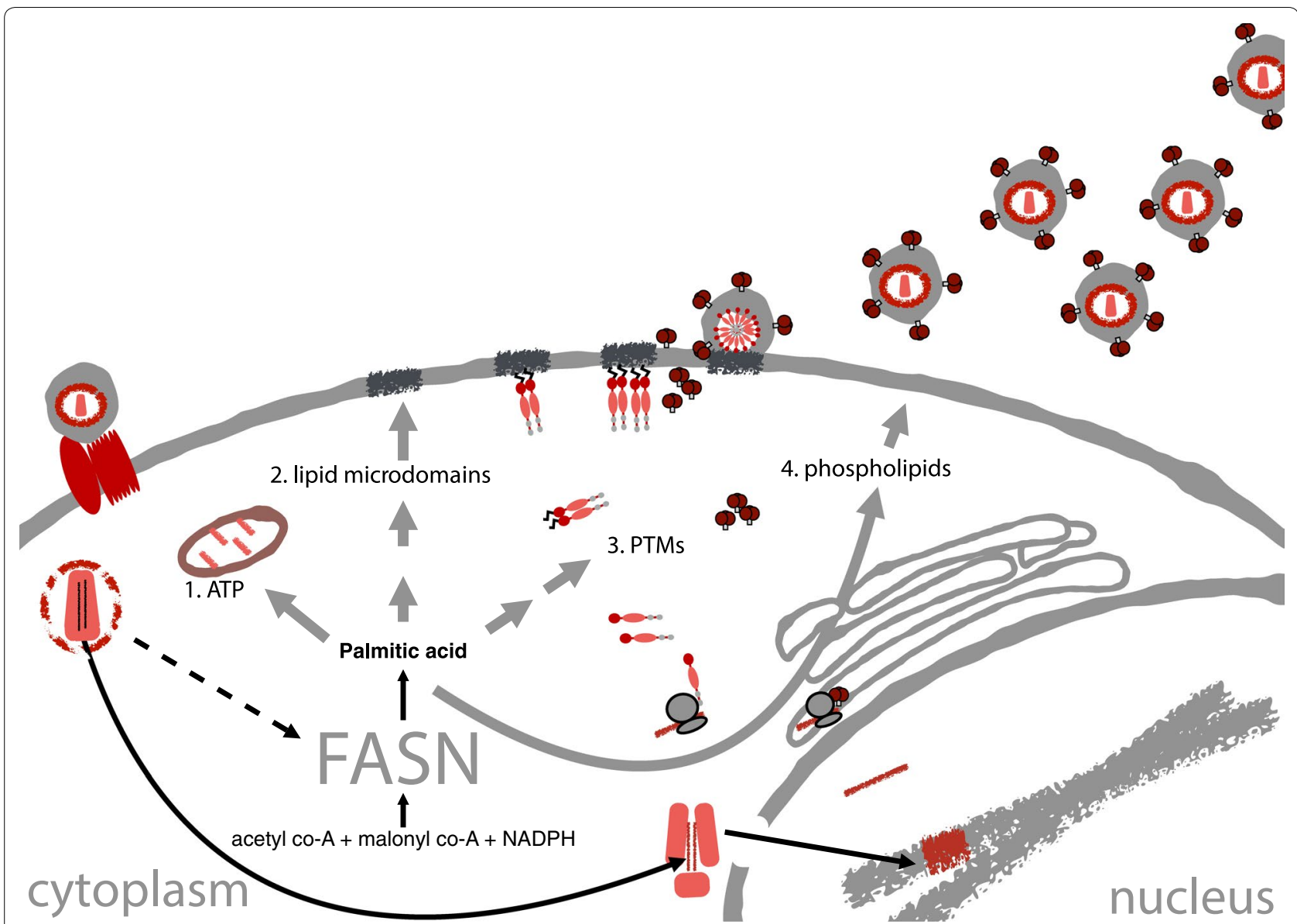

Fig. 7 Potential mechanisms linking FASN activity to HIV-1 replication. (1) Provision of fatty acids used for ATP production and energy homeostasis, (2) creation of lipid micro domains (rafts) favoring HIV-1 budding, (3) generation of fatty-acyl adducts (e.g. palmitate or myristate) for post-translational modification (PTM) of Env, Gag, Nef, or host proteins, (4) homeostatic replenishment of membrane lipids lost during viral budding

viral replication cycle. Based on these observations, we hypothesize that FASN is a novel host dependency factor and that inhibition of FASN activity has the potential to be exploited as an antiretroviral strategy.

\section{Methods}

\section{Reagents}

All chemicals were obtained from Sigma-Aldrich (St Louis, MO, USA). Cells were obtained from ATCC (Manassas, Virginia, USA), except TZM-bl cells, which were obtained from the NIH AIDS Reagent Program (submitted by Dr. John C. Kappes, Dr. Xiaoyun Wu and Tranzyme Inc.). A plasmid containing a HIV-1 provirus (pNL4-3) was obtained through the NIH AIDS Reagent Program from Dr. Malcolm Martin. A plasmid containing HIV-1 Tat was also obtained through the Aids Reagent Program (pcTAT). HIV-1 concentration was determined with a commercial p24 ELISA assay kit (Zeptometix, Buffalo, NY). Anti-HIV-1 p24 Monoclonal antibodies (71-31 or cat\# 530) and HIV-immunoglobulin
(HIVIG; cat\# 3957) were obtained from the AIDS Reagent Repository.

\section{Purinome capture}

ATP Sepharose was synthesized as described [54]. HIV$1_{\mathrm{NL4}-3}$ was produced in $293 \mathrm{~T}$ cells according to standard protocols [55]. TZM-bl cells were lysed at $4{ }^{\circ} \mathrm{C}$ in lysis buffer $(20 \mathrm{mM}$ hepes $\mathrm{pH} 7.4,1 \times$ complete protease inhibitors without EDTA (Roche, Indianapolis, IA), $120 \mathrm{mM}$ $\mathrm{NaCl}, 20 \mathrm{mM} \mathrm{MgCl}, 1 \mathrm{mM}$ DTT, $0.1 \% \mathrm{NP}-40$ ), centrifuged at $16,000 \times g$ for $10 \mathrm{~min}$ at $4{ }^{\circ} \mathrm{C}$, and the supernatant was loaded onto $100 \mu \mathrm{L}$ ATP Sepharose. ATP-Sepharose was incubated with cell lysate for $1 \mathrm{~h}$ at $4{ }^{\circ} \mathrm{C}$, washed $3 \times$ with low salt buffer $(50 \mathrm{mM}$ hepes $\mathrm{pH} 7.4,120 \mathrm{mM} \mathrm{NaCl}$, $20 \mathrm{mM} \mathrm{MgCl}, 1 \mathrm{mM}$ DTT), washed $2 \times$ with high salt buffer (low salt buffer with $300 \mathrm{mM} \mathrm{NaCl}$ [final]), then washed $2 \times$ with low salt buffer. Proteins were competed off the resin with $25 \mathrm{mM}$ ATP dissolved in low salt buffer, dialyzed to remove ATP, mixed with Laemmli sample buffer, and visualized by 1-D SDS PAGE. Gels were fixed 
and silver stained according to published protocols [17]. Individual proteins were manually excised from the gel, washed, dehydrated, and digested in porcine trypsin (Promega, Madison, WI), as described [56]. Peptides were spotted on a MALDI plate (matrix: alpha-cyano4-hydroxycinnamic acid (Aldrich Chemical Co. Milwaukee, WI) in a saturated solution of acetonitrile: $25 \mathrm{mM}$ aqueous ammonium citrate:trifuoroacetic acid (1:1:0.02, $v / v / v)$. MALDI-MS/MS data were acquired using the AB Sciex 5800 TOF/TOF Mass Spectrometer (AB Sciex, Framingham, MA). Peptide mass fingerprint and peptide sequence data were resolved by the SPROT(UNIPROT) and NCBI databases using the Mascot search engine.

\section{FASN visualization}

Equal amounts of cellular protein from TZM-bl cells with or without HIV-1 were separated on an 8\% SDS-PAGE gel, transferred to nitrocellulose, immune-blotted with anti-FASN (Abcam, Cambridge, MA), then anti-rabbit secondary Ab (Abcam, Cambridge, MA), and visualized with ECL detection reagent (GE Biosciences). To verify equal protein loading, the membrane was stripped and probed with anti-actin (Cell Signaling, Danvers, MA). Protein levels were quantified using ImageJ software (https://imagej.nih.gov). For immuno-fluorescence experiments, $1 \times 10^{5}$ TZM-bl cells were plated on sterilized coverslips, infected with $10 \mathrm{ng}(\mathrm{p} 24) / \mathrm{mL} \mathrm{HIV}^{-1}{ }_{\mathrm{NL4}-3}$ for $48 \mathrm{~h}$, washed twice with PBS, fixed with $4 \%$ paraformaldehyde, washed thrice with PBS, permeabilized with chilled methanol, washed thrice with PBS, blocked with $\mathrm{PBS}+1 \% \mathrm{BSA}$, incubated with anti-FASN primary Ab (Abcam, Cambridge, MA), and visualized with Alexa fluor-488-conjugated anti rabbit IgG (Life Technologies, New York, NY). Lysosomes were visualized using CD63 $\mathrm{Ab}$ ]Developmental studies hybridoma bank (DSHB), University of Iowa]; endoplasmic reticulum was stained with calreticulin Ab (DSHB, University of Iowa), and imaged with fluorescent secondary Ab (Alexa Fluor 594-anti mouse IgG, Life Technologies, New York, NY). Mitochondria were stained with mitotracker-red (Life Technologies, New York, NY). Coverslips were mounted onto fixed cells using Prolong Gold DAPI mounting medium (ThermoFisher, Waltham, MA) and observed on a FLUOVIEW Olympus microscope. Related images were collected for equivalent exposure times.

\section{Real time polymerase chain reaction (RT-PCR)}

TZM-bl cells infected with HIV-1 $1_{\mathrm{NL} 4-3}$ at $10 \mathrm{ng}(\mathrm{p} 24) / \mathrm{mL}$ were collected at intervals over $48 \mathrm{~h}$ of infection and total RNA was isolated using Qiagen RNeasy kit. Synthesis of cDNA was performed using oligo dT primer and Superscript III Reverse Transcriptase (Invitrogen, Carlsbad, CA). Real time PCR using SYBR green kit was performed according to manufacturer's instructions (BioRad, Hercules, CA). The FASN primers (sense, 5'-CCCACCTACGTACTGGCCTA-3'; antisense, 5'-CTTGGCCTTGGGTGTGTACT-3') were used to synthesize the PCR products. The $18 \mathrm{~s}$ ribosomal RNA subunit primers (sense, 5'-CAGCCACCCGAGATTGAGCA-3'; antisense, $5^{\prime}$-TAGTAGCGACGGGCGGTGTG-3') were used as controls to normalize FASN samples. PCR was run for 40 cycles, with 1 cycle consisting of $30 \mathrm{~s}$ at $95{ }^{\circ} \mathrm{C}, 30 \mathrm{~s}$ at $55^{\circ} \mathrm{C}$, and 30 s at $72{ }^{\circ} \mathrm{C}$.

\section{SiRNA knockdown of FASN}

ON-TARGET plus SMART pool of four siRNAs targeted against human FASN (FASN) (L-003954-00-0005) and ON-TARGET plus non-targeting (NT) control siRNA (D-001810-01-05) were purchased from Dharmacon (Thermo Fisher Scientific, Waltham, MA). TZM-bl cells were transfected either with $200 \mathrm{nM}$ FASN-targeting siRNA or $200 \mathrm{nM}$ NT siRNA using Trans-IT transfection reagent (Mirus Bio LLC, Madison, WI). After 48 h, cells were infected with $10 \mathrm{ng}(\mathrm{p} 24) / \mathrm{mL} \mathrm{HIV}-1_{\mathrm{NL} 4-3}$, incubated for $24 \mathrm{~h}$, medium was replenished with fresh medium, and incubated for additional $24 \mathrm{~h}$. Supernatants were collected for HIV-1 p24 ELISA and cells were washed with PBS and saved for Western blotting.

\section{Fatty acid analysis and quantification}

TZM-bl cells were infected with 0,20 , or $40 \mathrm{ng}$ (p24)/ $\mathrm{mL} \mathrm{HIV-1} 1_{\mathrm{NL4}-3}$ and total fatty acids were extracted from an equivalent number of cells using a modified version of the Bligh and Dyer protocol [57]. This consisted of sequential extractions with chloroform:methanol (2:1, 1:1 and 1:2, $v / v)$, and chloroform:methanol:water $(10: 10: 3, v / v / v)$. Fatty acid methyl esters were generated by methanolysis with $3 \mathrm{~N}$ methanolic- $\mathrm{HCl}\left(85^{\circ} \mathrm{C}\right.$ overnight) followed by trimethylsilylation with Tri-Sil reagent (Thermo Scientific, Waltham, MA). Heptadecanoic acid $(17: 0)$ was used as internal standard. Samples were dissolved in hexane prior to injection on a Thermo Scientific Trace GC-MS ULTRA/DSQII with a Rtx-5MS column $(30 \mathrm{~m} \times 0.25 \mathrm{~mm}$ internal diameter, $0.25 \mu \mathrm{m}$ film thickness, Restek Corporation, Bellefonte, PA). Instrument settings included an internal temperature of $150{ }^{\circ} \mathrm{C}$ for 3 min, increasing to $200{ }^{\circ} \mathrm{C}$ at $2{ }^{\circ} \mathrm{C} / \mathrm{min}$ and to $250{ }^{\circ} \mathrm{C}$ at $40^{\circ} \mathrm{C} / \mathrm{min}$ holding for $4 \mathrm{~min}$.

\section{TZM-bl infection assays}

HIV-1 $1_{\text {NL4-3 }}$ was added to $3 \times 10^{4}$ TZM-bl cells at $10 \mathrm{ng}$ (p24)/mL equivalents in the presence of $7.5 \mu \mathrm{g} / \mathrm{mL}$ DEAE dextran. Fasnall or C75 were serially diluted in DMSO, and all assays had a final DMSO concentration of $\leq 1 \%$. HIV-infected cells were incubated at $37{ }^{\circ} \mathrm{C}$ in $5 \% \mathrm{CO}_{2}$ for $24 \mathrm{~h}$, washed with PBS, fresh medium was 
added, and cell were incubated for another $24 \mathrm{~h}$. HIV-1 replication was assessed by quantitative p24 ELISA. Nevirapine (positive control) was obtained from the AIDS Reagent Repository.

\section{Primary cell infection}

PBMCs were isolated by Ficoll-Paque centrifugation, stimulated in complete RPMI-1640 medium (Gibco, Carlsbad, CA) containing 10\% FBS, $100 \mu \mathrm{g} / \mathrm{mL}$ penicillin/streptomycin and supplemented with $5 \mu \mathrm{g} / \mathrm{mL}$ phytohemagglutinin (PHA; Gibco) for $48 \mathrm{~h}$, and maintained thereafter in complete RPMI-1640 medium supplemented with $20 \mathrm{U} / \mathrm{mL}$ of interleukin-2 (Gibco). PBMCs were seeded in 24 -well plate $\left(2 \times 10^{5}\right.$ cells/well $)$ and triplicate wells were treated with indicated concentrations of C75, FASNALL, or with DMSO, and subsequently infected with $10 \mathrm{ng}(\mathrm{p} 24) / \mathrm{mL}$ equivalents of $\mathrm{HIV}-1_{\mathrm{NL4}-3}$. Cells were washed $24 \mathrm{~h}$ post infection, and supernatants were collected and p24 content analyzed by quantitative ELISA (Zeptometrix, Buffalo, NY). PBMC viability was assessed by flow cytometry using propidium iodine (PI) exclusion (BD Pharmingen).

\section{Authors' contributions}

Planned experiments and generated data: MMK, ANR, MB, JA, DL. All authors analyzed data and interpreted results. TAH and JJK wrote the first draft of the article. All authors read and approved the final manuscript.

\section{Author details}

${ }^{1}$ Department of Microbial Infection and Immunity, Center for Microbial Interface Biology, The Ohio State University, Columbus, OH, USA. ${ }^{2}$ Department of Pharmacology and Cancer Biology, Duke University School of Medicine, C118 LSRC, Box 3813, Durham, NC 27710, USA. ${ }^{3}$ Division of Medical Laboratory Science, School of Health and Rehabilitation Sciences, The Ohio State University, Columbus, OH, USA. ${ }^{4}$ Department of Microbiology, Center for Retrovirus Research, The Ohio State University, 476 Biological Sciences Building, 484 W. 12th Avenue, Columbus, OH 43210, USA. ${ }^{5}$ Present Address: Promega Corporation, 2800 Woods Hollow Rd, Madison, WI 53711-5399, USA. ${ }^{6}$ Present Address: Texas Biomedical Research Institute, San Antonio, Texas, USA.

\section{Acknowledgements}

We thank Dr. Jacob Yount for a critical reading of this manuscript. Requests for Fasnall should be directed to Dr. T.A. Haystead. Data from this manuscript was presented in part at the $42^{\text {nd }}$ annual meeting on Retroviruses at Cold Spring Harbor Laboratory.

\section{Competing interests}

Drs. Alwarawrah, Hughes, Haystead, and Kwiek have filed a patent on Fasnall. Dr. Ratcliff is currently an employee of Promega Corporation; her contributions to this manuscript occurred while she was an employee of The Ohio State University.

\section{Availability of data and materials}

All data generated or analyzed during this study are included in this published article.

\section{Consent for publication}

Not applicable.

\section{Ethics approval and consent to participate}

Primary cell experiments were approved by The Ohio State University Institutional Review Board, protocol \#2014H0001. All blood donors provided informed consent.

\section{Funding}

This research was supported in part by NIH awards Al090644 (JJK, TAH), R00HL108743 (NTF) and R56HL126563 (NTF), and an OSU Cancer Center Pelotonia Fellowship (ANR). The content of this article is solely the responsibility of the authors and it does not necessarily represent the official views of the $\mathrm{NIH}$ or the Pelotonia Fellowship Program.

\section{Publisher's Note}

Springer Nature remains neutral with regard to jurisdictional claims in published maps and institutional affiliations.

Received: 7 February 2017 Accepted: 14 September 2017

Published online: 29 September 2017

\section{References}

1. Park RJ, Wang T, Koundakjian D, Hultquist JF, Lamothe-Molina P, Monel B, Schumann K, Yu H, Krupzcak KM, Garcia-Beltran W, Piechocka-Trocha A, Krogan NJ, Marson A, Sabatini DM, Lander ES, Hacohen N, Walker BD. A genome-wide CRISPR screen identifies a restricted set of HIV host dependency factors. Nat Genet. 2017;49(2):193-203. doi:10.1038/ng.3741.

2. Krishnan MN, Ng A, Sukumaran B, Gilfoy FD, Uchil PD, Sultana H, Brass AL, Adametz R, Tsui M, Qian F, Montgomery RR, Lev S, Mason PW, Koski RA, Elledge SJ, Xavier RJ, Agaisse H, Fikrig E. RNA interference screen for human genes associated with West Nile virus infection. Nature. 2008;455:242-5.

3. Sessions OM, Barrows NJ, Souza-Neto JA, Robinson TJ, Hershey CL, Rodgers MA, Ramirez JL, Dimopoulos G, Yang PL, Pearson JL, Garcia-Blanco MA. Discovery of insect and human dengue virus host factors. Nature. 2009:458:1047-50.

4. Tai AW, Benita Y, Peng LF, Kim SS, Sakamoto N, Xavier RJ, Chung RT. A functional genomic screen identifies cellular cofactors of hepatitis $C$ virus replication. Cell Host Microbe. 2009;5:298-307.

5. Stertz S, Shaw ML. Uncovering the global host cell requirements for influenza virus replication via RNAi screening. Microbes Infect. 2011;13:516-25.

6. Brass AL, Dykxhoorn DM, Benita Y, Yan N, Engelman A, Xavier RJ, Lieberman J, Elledge SJ. Identification of host proteins required for HIV infection through a functional genomic screen. Science. 2008;319:921-6.

7. Bushman FD, Malani N, Fernandes J, D'Orso I, Cagney G, Diamond TL, Zhou H, Hazuda DJ, Espeseth AS, Konig R, Bandyopadhyay S, Ideker T, Goff SP, Krogan NJ, Frankel AD, Young JA, Chanda SK. Host cell factors in HIV replication: meta-analysis of genome-wide studies. PLoS Pathog. 2009;5:e1000437.

8. Goff SP. Knockdown screens to knockout HIV-1. Cell. 2008;135:417-20.

9. Konig R, Zhou Y, Elleder D, Diamond TL, Bonamy GM, Irelan JT, Chiang CY, Tu BP, De Jesus PD, Lilley CE, Seidel S, Opaluch AM, Caldwell JS, Weitzman MD, Kuhen KL, Bandyopadhyay S, Ideker T, Orth AP, Miraglia LJ, Bushman FD, Young JA, Chanda SK. Global analysis of host-pathogen interactions that regulate early-stage HIV-1 replication. Cell. 2008;135:49-60.

10. Zhou H, Xu M, Huang Q, Gates AT, Zhang XD, Castle JC, Stec E, Ferrer M, Strulovici B, Hazuda DJ, Espeseth AS. Genome-scale RNAi screen for host factors required for HIV replication. Cell Host Microbe. 2008;4:495-504.

11. Dorr P, Westby M, Dobbs S, Griffin P, Irvine B, Macartney M, Mori J, Rickett G, Smith-Burchnell C, Napier C, Webster R, Armour D, Price D, Stammen B, Wood A, Perros M. Maraviroc (UK-427,857), a potent, orally bioavailable, and selective small-molecule inhibitor of chemokine receptor CCR5 with broad-spectrum anti-human immunodeficiency virus type 1 activity. Antimicrob Agents Chemother. 2005;49:4721-32.

12. Howe MK, Speer BL, Hughes PF, Loiselle DR, Vasudevan S, Haystead TA. An inducible heat shock protein 70 small molecule inhibitor demonstrates anti-dengue virus activity, validating $\mathrm{Hsp} 70$ as a host antiviral target. Antiviral Res. 2016;130:81-92.

13. Rathore AP, Haystead T, Das PK, Merits A, Ng ML, Vasudevan SG. Chikungunya virus nsP3 \& nsP4 interacts with HSP-90 to promote virus replication: HSP-90 inhibitors reduce CHIKV infection and inflammation in vivo. Antiviral Res. 2014;103:7-16. 
14. Taguwa S, Maringer K, Li X, Bernal-Rubio D, Rauch JN, Gestwicki JE, Andino R, Fernandez-Sesma A, Frydman J. Defining Hsp70 subnetworks in dengue virus replication reveals key vulnerability in flavivirus infection. Cell. 2015;163:1108-23.

15. Alwarawrah $Y$, Hughes $P$, Loiselle D, Carlson DA, Darr DB, Jordan JL, Xiong J, Hunter LM, Dubois LG, Thompson JW, Kulkarni MM, Ratcliff AN, Kwiek JJ, Haystead TA. Fasnall, a selective FASN inhibitor, shows potent anti-tumor activity in the MMTV-Neu model of HER2(+) breast cancer. Cell Chem Biol. 2016;23:678-88.

16. Fadden $P$, Huang $K H$, Veal JM, Steed PM, Barabasz AF, Foley B, Hu M, Partridge JM, Rice J, Scott A, Dubois LG, Freed TA, Silinski MA, Barta TE, Hughes PF, Ommen A, Ma W, Smith ED, Spangenberg AW, Eaves J, Hanson GJ, Hinkley L, Jenks M, Lewis M, Otto J, Pronk GJ, Verleysen K, Haystead TA, Hall SE. Application of chemoproteomics to drug discovery: identification of a clinical candidate targeting hsp90. Chem Biol. 2010;17:686-94

17. Graves PR, Kwiek JJ, Fadden P, Ray R, Hardeman K, Coley AM, Foley M, Haystead TA. Discovery of novel targets of quinoline drugs in the human purine binding proteome. Mol Pharmacol. 2002;62:1364-72.

18. Anderson I, Low JS, Weston S, Weinberger M, Zhyvoloup A, Labokha AA, Corazza G, Kitson RA, Moody CJ, Marcello A, Fassati A. Heat shock protein 90 controls HIV-1 reactivation from latency. Proc Natl Acad Sci USA. 2014;111:E1528-37.

19. Roesch F, Meziane O, Kula A, Nisole S, Porrot F, Anderson I, Mammano F, Fassati A, Marcello A, Benkirane M, Schwartz O. Hyperthermia stimulates HIV-1 replication. PLoS Pathog. 2012;8:e1002792.

20. Vozzolo L, Loh B, Gane PJ, Tribak M, Zhou L, Anderson I, Nyakatura E, Jenner RG, Selwood D, Fassati A. Gyrase B inhibitor impairs HIV-1 replication by targeting Hsp90 and the capsid protein. J Biol Chem. 2010;285:39314-28

21. Weiss L, Hoffmann GE, Schreiber R, Andres H, Fuchs E, Korber E, Kolb HJ. Fatty-acid biosynthesis in man, a pathway of minor importance. Purification, optimal assay conditions, and organ distribution of fatty-acid synthase. Biol Chem Hoppe Seyler. 1986;367:905-12.

22. Heaton NS, Perera R, Berger KL, Khadka S, Lacount DJ, Kuhn RJ, Randall $\mathrm{G}$. Dengue virus nonstructural protein 3 redistributes fatty acid synthase to sites of viral replication and increases cellular fatty acid synthesis. Proc Natl Acad Sci USA. 2010;107:17345-50.

23. Huang JT, Tseng CP, Liao MH, Lu SC, Yeh WZ, Sakamoto N, Chen CM, Cheng JC. Hepatitis $C$ virus replication is modulated by the interaction of nonstructural protein NS5B and fatty acid synthase. J Virol. 2013:87:4994-5004

24. Martin-Acebes MA, Blazquez AB, Jimenez de Oya N, Escribano-Romero $E$, Saiz JC. West Nile virus replication requires fatty acid synthesis but is independent on phosphatidylinositol-4-phosphate lipids. PLoS ONE. 2011;6:e24970

25. Liu H, Liu JY, Wu X, Zhang JT. Biochemistry, molecular biology, and pharmacology of fatty acid synthase, an emerging therapeutic target and diagnosis/prognosis marker. Int J Biochem Mol Biol. 2010;1:69-89.

26. Greseth MD, Traktman P. De novo fatty acid biosynthesis contributes significantly to establishment of a bioenergetically favorable environment for vaccinia virus infection. PLoS Pathog. 2014;10:e1004021.

27. Yang W, Hood BL, Chadwick SL, Liu S, Watkins SC, Luo G, Conrads TP, Wang T. Fatty acid synthase is up-regulated during hepatitis $C$ virus infection and regulates hepatitis $C$ virus entry and production. Hepatology. 2008:48:1396-403.

28. LiY, Webster-Cyriaque J, Tomlinson CC, Yohe M, Kenney S. Fatty acid synthase expression is induced by the Epstein-Barr virus immediateearly protein BRLF1 and is required for lytic viral gene expression. J Virol. 2004;78:4197-206.

29. Wilsky S, Sobotta K, Wiesener N, Pilas J, Althof N, Munder T, Wutzler P, Henke A. Inhibition of fatty acid synthase by amentoflavone reduces coxsackievirus B3 replication. Arch Virol. 2012;157:259-69.

30. Munger J, Bennett BD, Parikh A, Feng XJ, McArdle J, Rabitz HA, Shenk T, Rabinowitz JD. Systems-level metabolic flux profiling identifies fatty acid synthesis as a target for antiviral therapy. Nat Biotechnol. 2008;26:1179-86

31. Kuhajda FP. Fatty-acid synthase and human cancer: new perspectives on its role in tumor biology. Nutrition. 2000;16:202-8.

32. Chan EY, Qian WJ, Diamond DL, Liu T, Gritsenko MA, Monroe ME, Camp DG, Smith RD, Katze MG. Quantitative analysis of human immunodeficiency virus type 1-infected CD4 + cell proteome: dysregulated cell cycle progression and nuclear transport coincide with robust virus production. J Virol. 2007;81:7571-83.

33. Rasheed S, Yan JS, Lau A, Chan AS. HIV replication enhances production of free fatty acids, low density lipoproteins and many key proteins involved in lipid metabolism: a proteomics study. PLOS ONE. 2008:3:e3003.

34. Aragones G, Alonso-Villaverde C, Oliveras-Ferraros C, Beltran-Debon R, Rull A, Rodriguez-Sanabria F, Camps J, Martin AV, Menendez JA, Joven J. Infection with HIV and HCV enhances the release of fatty acid synthase into circulation: evidence for a novel indicator of viral infection. BMC Gastroenterol. 2010;10:92.

35. Wakil SJ. Fatty acid synthase, a proficient multifunctional enzyme. Biochemistry. 1989;28:4523-30.

36. Menendez JA, Lupu R. Fatty acid synthase and the lipogenic phenotype in cancer pathogenesis. Nat Rev Cancer. 2007;7:763-77.

37. Ono A, Freed EO. Plasma membrane rafts play a critical role in HIV-1 assembly and release. Proc Natl Acad Sci USA. 2001:98:13925-30.

38. Dick RA, Goh SL, Feigenson GW, Vogt VM. HIV-1 Gag protein can sense the cholesterol and acyl chain environment in model membranes. Proc Natl Acad Sci USA. 2012;109:18761-6.

39. Llewellyn GN, Grover JR, Olety B, Ono A. HIV-1 Gag associates with specific uropod-directed microdomains in a manner dependent on its MA highly basic region. J Virol. 2013;87:6441-54.

40. Olety B, Veatch SL, Ono A. Phosphatidylinositol-(4,5)-bisphosphate acyl chains differentiate membrane binding of HIV-1 Gag from that of the phospholipase C 81 pleckstrin homology domain. J Virol. 2015;89:7861-73

41. Bryant M, Ratner L. Myristoylation-dependent replication and assembly of human immunodeficiency virus 1. Proc Natl Acad Sci USA. 1990;87:523-7.

42. Li H, Dou J, Ding L, Spearman P. Myristoylation is required for human immunodeficiency virus type 1 Gag-Gag multimerization in mammalian cells. J Virol. 2007:81:12899-910.

43. Lindwasser OW, Resh MD. Myristoylation as a target for inhibiting HIV assembly: unsaturated fatty acids block viral budding. Proc Natl Acad Sci USA. 2002;99:13037-42.

44. Pal R, Gallo RC, Sarngadharan MG. Processing of the structural proteins of human immunodeficiency virus type 1 in the presence of monensin and cerulenin. Proc Natl Acad Sci USA. 1988;85:9283-6.

45. Rousso I, Mixon MB, Chen BK, Kim PS. Palmitoylation of the HIV-1 envelope glycoprotein is critical for viral infectivity. Proc Natl Acad Sci USA. 2000;97:13523-5.

46. Chan WE, Lin HH, Chen SS. Wild-type-like viral replication potential of human immunodeficiency virus type 1 envelope mutants lacking palmitoylation signals. J Virol. 2005;79:8374-87.

47. Gottlinger HG, Sodroski JG, Haseltine WA. Role of capsid precursor processing and myristoylation in morphogenesis and infectivity of human immunodeficiency virus type 1. Proc Natl Acad Sci USA. 1989;86:5781-5.

48. Baugh JM, Garcia-Rivera JA, Gallay PA. Host-targeting agents in the treatment of hepatitis C: a beginning and an end? Antiviral Res. 2013;100:555-61.

49. Puig T, Aguilar H, Cufi S, Oliveras G, Turrado C, Ortega-Gutierrez S, Benhamu B, Lopez-Rodriguez ML, Urruticoechea A, Colomer R. A novel inhibitor of fatty acid synthase shows activity against HER2+ breast cancer xenografts and is active in anti-HER2 drug-resistant cell lines. Breast Cancer Res. 2011;13:R131.

50. Yoshii Y, Furukawa T, Oyama N, Hasegawa Y, Kiyono Y, Nishii R, Waki A, Tsuji AB, Sogawa C, Wakizaka H, Fukumura T, Yoshii H, Fujibayashi Y, Lewis JS, Saga T. Fatty acid synthase is a key target in multiple essential tumor functions of prostate cancer: uptake of radiolabeled acetate as a predictor of the targeted therapy outcome. PLOS ONE. 2013;8:e64570.

51. Chirala SS, Chang H, Matzuk M, Abu-Elheiga L, Mao J, Mahon K, Finegold M, Wakil SJ. Fatty acid synthesis is essential in embryonic development: fatty acid synthase null mutants and most of the heterozygotes die in utero. Proc Natl Acad Sci USA. 2003;100:6358-63.

52. Chakravarthy MV, Pan Z, Zhu Y, Tordjman K, Schneider JG, Coleman T, Turk J, Semenkovich CF. "New" hepatic fat activates PPARalpha to maintain glucose, lipid, and cholesterol homeostasis. Cell Metab. 2005;1:309-22.

53. Schneider JG, Yang Z, Chakravarthy MV, Lodhi IJ, Wei X, Turk J, Semenkovich CF. Macrophage fatty-acid synthase deficiency decreases dietinduced atherosclerosis. J Biol Chem. 2010;285:23398-409. 
54. Haystead CM, Gregory P, Sturgill TW, Haystead TA. Gamma-phosphatelinked ATP-sepharose for the affinity purification of protein kinases.

Rapid purification to homogeneity of skeletal muscle mitogen-activated protein kinase kinase. Eur J Biochem. 1993;214:459-67.

55. Russell ES, Kwiek JJ, Keys J, Barton K, Mwapasa V, Montefiori DC, Meshnick SR, Swanstrom R. The genetic bottleneck in vertical transmission of subtype C HIV-1 is not driven by selection of especially neutralization-resistant virus from the maternal viral population. J Virol. 2011;85:8253-62.
56. Graves PR, Haystead TA. Molecular biologist's guide to proteomics. Microbiol Mol Biol Rev. 2002;66:39-63 (table of contents)

57. Bligh EG, Dyer WJ. A rapid method of total lipid extraction and purification. Can J Biochem Physiol. 1959;37:911-7.

\section{Submit your next manuscript to BioMed Central and we will help you at every step:}

- We accept pre-submission inquiries

- Our selector tool helps you to find the most relevant journal

- We provide round the clock customer support

- Convenient online submission

- Thorough peer review

- Inclusion in PubMed and all major indexing services

- Maximum visibility for your research

Submit your manuscript at

www.biomedcentral.com/submit 